\title{
FOURIER TRANSFORMS OF LIPSCHITZ FUNCTIONS ON THE HYPERBOLIC PLANE $H^{2}$
}

\author{
M.S. YOUNIS \\ Department of Mathematics \\ Yarmouk University \\ Irbid, JORDAN
}

(Received April 24, 1996)

\begin{abstract}
The purpose of the present work is to study the order of magnitude of the Fourier transforms $\hat{f}(\lambda)$ for large $\lambda$ of complex-valued functions $f(z)$ satisfying certain Lipschitz conditions in the non-Euclidean hyperbolic plane $H^{2}$.
\end{abstract}

KEY WORDS AND PHRASES: Fourier transforms, Lipschitz functions, absolute convergence of Fourier transforms.

1991 AMS SUBJECT CLASSIFICATION CODES: Primary 42B10.

\section{INTRODUCTION}

The relation between smoothness conditions imposed on functions $f(x)$ and the behavior of its Fourier transforms $\widehat{f}$ near infinity is well known in the literature.

In fact, the Fourier transforms of Lipschitz functions defined on various domains have been extensively studied over the last decades. The purpose of the present research is to trace the behavior of the Fourier transforms of complex-valued functions satisfying Lipschitz conditions in the hyperbolic plane $H^{2}$. This will pave the ground for handling the transforms of Lipschitz functions defined on other domains such as SL(2.R) and SL(2.C) in particular.

\section{DEFINITIONS AND NOTATIONS}

Our main reference on the Fourier analysis on $\mathrm{H}^{2}$ is the book by Helgason ([2], p. 29 infra); reference [7] will be consulted (especially Chapter 10) as well. In the beginning we would like to make clear an idea which will be frequently encountered in the sequel. Let $D$ be the open disc $|z|<1$ in the plane $R^{2}$. Then a horocycle in $D$ is a circle tangential to the boundary $B=\partial D$ at a certain point $b$. This horocycle is denoted by $\xi$. If $z$ is a point on $\xi$, the distance $d(0, z)$ from the origin 0 of $D$ to $z$ is denoted by $\langle z, b\rangle$. Let $d(0, z)=r$. Then

$$
r=d(0, z)=\frac{1}{2} \log \frac{1+|z|}{1-|z|}
$$

This gives $|z|=\tanh r$. We indicate here that $\tanh r$ is of the order of $r$ near $r=0$ and that it approaches 1 as $r$ goes to infinity. These two limits will occur frequently in due course. In this section we introduce the basic definitions necessary for the proof of the main theorems. Other definitions will be given when they are needed. Thus we start with

DEFINITION 2.1. Let $f(z)$ be a complex-valued function defined on the unit disc $D$ when endowed with the Riemannian structure. Then $f(z)$ is said to belong to the $\operatorname{Lipschitz}$ class $\operatorname{Lip}(\alpha, 2)$ if

$$
\|f(z+h)-f(z)\|_{2}=O\left(|h|^{\alpha}\right)
$$


as $h \rightarrow 0,0<\alpha<1$. Where $\|\cdot\|_{2}$ is the usual $L^{2}$ norm. The generalization of this definition for higher differences $\triangle_{|h|}^{k} f(z)$ of order $k$ in step $h$ takes the form

$$
\left\|\triangle_{|h|}^{k} f(z)\right\|_{2}=O\left(|h|^{\alpha}\right) \quad 0<\alpha \leq k+1 .
$$

DEFINITION 2.2 ([2], p. 33). If $f(z)$ is a complex-valued function on $D$, its Fourier transform is defined by

$$
\widehat{f}(\lambda, b)=\int_{\mathcal{D}} f(z) e^{(-\imath \lambda+1)(z, b)} d z
$$

for all $\lambda \in C, b \in B$ for which this integral exists. The Parseval's identity in this case is written as

$$
\int_{D}|f(z)|^{2} d z=\frac{1}{2 \pi} \int_{0}^{\infty} \int_{B}|\widehat{f}|^{2} \lambda \tanh \left(\frac{\pi \lambda}{2}\right) d \lambda d b .
$$

\section{MAIN THEOREMS}

Our main result may be viewed as the non-Euclidean analogue of the following theorem.

THEOREM 3.1 ([5], Theorem 85). Let $f(x)$ belong to $L^{2}(R)$. Then the conditions

$$
\|f(x+h)-f(x)\|_{2}=O\left(h^{\alpha}\right)
$$

as $h \rightarrow 0,0<\alpha<1$ and

$$
\left[\int_{-\infty}^{-X}+\int_{X}^{\infty}\right]|\widehat{f}|^{2} d x=O\left(X^{-2 \alpha}\right)
$$

as $X \rightarrow \infty$ are equivalent. The main theorem of this section is stated as

THEOREM 3.2. Let $f(z)$ be a complex-valued function on the unit disc $D$. Then the conditions

$$
\|f(z+h)-f(z)\|_{2}=O\left(|h|^{\alpha}\right)
$$

as $|h| \rightarrow 0,0<\alpha<1$ and

$$
\int_{\lambda}^{\infty} \int_{B}|\widehat{f}|^{2} d \lambda d b=O\left(\lambda^{-2 a-1}\right)
$$

as $\lambda \rightarrow \infty$ are equivalent.

PROOF. By definition of $\widehat{f}(\lambda, b)$ it is easily seen that the transform of $(f(z+h)-f(z))$ is given by

$$
\left(e^{(-i \lambda+1)(-h, b)}-1\right) \hat{f}(\lambda, b)
$$

observe that $\langle-h, b\rangle=\langle h, b\rangle=d(0, h)=r$ and hence $r \rightarrow 0$ with $|h|$ where $|h|=\tanh r$. So, the factor $e^{\langle h, b\rangle}=e^{r}$ tends to one as $r$ goes to zero and therefore can be suppressed without harm. Also, since $\lambda \in R^{+}$in the right side of the Parseval's identity ([7], p. 376), (10) could be simplified so that the transform of $|f(z+h)-f(z)|$ is given as $\left|2 \sin \frac{r \lambda}{2} \hat{f}\right|$. Thus

$$
\int_{0}^{\infty} \int_{B}\left|2 \sin \frac{r \lambda}{2} \widehat{f}\right|^{2} \lambda \tanh \left(\frac{\pi \lambda}{2}\right) d \lambda d b=O(\tanh r)^{2 \alpha}=O\left(r^{2 \alpha}\right) .
$$

Observe that $\tanh \left(\frac{\pi \lambda}{2}\right)$ tends to be a bounded constant for large $\lambda$. This yields (as in the proof of Theorem 3.1)

$$
\int_{\frac{1}{r}}^{\frac{2}{r}} \int_{B}|\widehat{f}|^{2} \lambda d \lambda d b=O\left(r^{2 \alpha}\right)=O\left(\lambda^{-2 \alpha}\right)
$$

equivalently 


$$
\int_{\frac{1}{r}}^{\frac{2}{r}} \int_{B}|\widehat{f}|^{2} d \lambda d b=O\left(\lambda^{-2 \alpha-1}\right) .
$$

From this one obtains

$$
\int_{\lambda}^{\infty} \int_{B}|\widehat{f}|^{2} d \lambda d b=\int_{\lambda}^{2 \lambda} \int_{B}+\int_{2 \lambda}^{4 \lambda} \int_{B}+\cdots+\cdots=O\left(\lambda^{-2 \alpha-1}\right)
$$

as $\lambda$ tends to infinity, this proves the first part of the theorem.

On the other hand, given (12), then by following the reverse argument as in the proof of Theorem 3.1 one can arrive easily at the estimate

$$
\begin{gathered}
\|f(z+h)-f(z)\|_{2}^{2}=O\left(r^{2 \alpha}\right)=O\left(\tanh r^{2 \alpha}\right) \\
=O\left(|h|^{2 \alpha}\right)
\end{gathered}
$$

as $|h| \rightarrow 0$ and the proof is complete.

REMARK 3.3. We indicate here that if the $k$-th difference $\triangle_{|h|}^{k} f(z)$ is employed in Definition 2.2 of the Lipschitz condition, then this would result in the appearance of the factor $|r \lambda|^{2 k}$ in place of $|r \lambda|^{2}$ in the estimates given by (10), (11), but this will not affect the final conclusion of Theorem 3.2. This is due to the way in which $r$ and $\lambda$ are tied up in their variation.

REMARK 3.4. Applying Hölder's inequality to (12) for $\beta \leq 2$ we get

$$
\begin{aligned}
\int_{\lambda}^{\infty} \int_{B}|\widehat{f}|^{\beta} d \lambda d b & =\left[O\left(\lambda^{-2 \alpha-1}\right)\right]^{\frac{\beta}{2}}\left[\int_{\lambda}^{\infty} \int_{B} d \lambda d b\right]^{1-\frac{\beta}{2}} \\
& =O\left(\lambda^{1-\alpha \beta-\beta}\right) .
\end{aligned}
$$

It is clear that the integral $\int_{B} d b=1$ when the normalized Haar measure is in action. The last quantity is bounded as $\lambda \rightarrow \infty$ if $1-\alpha \beta-\beta<0$, so that $\frac{1}{1+\alpha}<\beta \leq 2$, giving the condition $\alpha>0$ for $\beta=1$. This shows that the Fourier transform $\hat{f}(\lambda, b)$ of $f(z)$ converges absolutely for any $\alpha$ greater than zero. This reflects the strength of the conclusion in Theorem 3.2 in contrast to those of similar theorems proved for Lipschitz functions in $R, R^{2}$ and $T$ for example. This is mainly due to the presence of the weight $\lambda$ in the inversion formula as well as in one side of Parseval's identity. Had it not been for this extra factor in the present situation, the conclusion of Theorem 3.2 would have been exactly the same as that of Theorem 3.1 as far as the order of magnitude and the absolute convergence of $\widehat{f}$ are concerned. We shall encounter the same situation when dealing with the spherical Fourier transforms of Lipschitz functions in $H^{2}$ which is the subject matter of the next section.

\section{SPHERICAL TRANSFORMS IN $\boldsymbol{H}^{2}$}

In this section we prove an analogue of Theorem 3.2 for the spherical Fourier transforms of Lipschitz functions. These are related to spherical functions which (by their very nature) are radial eigenfunctions of the Laplacian on the unit disc (see [2], pp. 38, 39 for details). They are defined by

$$
\phi_{\lambda}(z)=\int_{B} e^{(-\imath \lambda+1)(z, b)} d b
$$

If $f(z)$ is a radial complex-valued function in $D$, then its spherical-Fourier transform is given by

$$
\widehat{f}(\lambda)=\int_{D} f(z) \phi_{-\lambda}(z) d z
$$

with this in hand, we state the following

THEOREM 4.1. Let $f(z)$ be radial in $D$. Then the conclusion of Theorem 3.2 holds with the integral 


$$
\int_{\lambda}^{\infty} \int_{B}|\widehat{f}|^{2} d \lambda d b
$$

being replaced with

$$
\int_{\lambda}^{\infty}|\widehat{f}|^{2} d \lambda
$$

PROOF. The proof will be carried briefly; we mainly point out the necessary modifications which will suit the present situation. One essential aspect here is the presence of the Harish-Chandra c-function both in the inversion formula as well as in the Parseval's identity. Thus one has

$$
C(\lambda)=\frac{1}{\sqrt{\pi}} \Gamma\left(\frac{i \lambda}{2}\right) / \Gamma\left(\frac{1}{2}(i \lambda+1)\right), \quad \lambda \in C
$$

whereas for $\lambda \in R$

$$
|C(\lambda)|^{-2}=\frac{1}{2 \pi} \lambda \tanh \left(\frac{\pi \lambda}{2}\right) .
$$

The Parseval's identity reads in this case

$$
\int_{D}|f(z)|^{2} d z=\frac{1}{2 \pi} \int_{0}^{\infty}|\widehat{f}|^{2}|C(\lambda)|^{-2} d \lambda .
$$

The transform of $f(z+h)$ is

$$
\begin{aligned}
\int_{D} f(z+h) \phi_{-\lambda}(z) d z & =\int_{D} f(w)\left[\int_{\mathcal{B}} \mathrm{e}^{(-i \lambda+1)\langle w-h, b\rangle} d b\right] d w \\
& =e^{(-i \lambda+1)\langle h, b\rangle} \widehat{f}(\lambda) .
\end{aligned}
$$

Taking into account the behavior of $e^{(h, b)}=e^{r}$ as $r$ tends to zero and that $|C(\lambda)|^{-2}=O(\lambda)$ for large $\lambda$, the proof of. Theorem 3.2 could be applied (almost word for word) to yield the required result of the theorem

We remark at this point that the degree of similarity between the last two theorems is not all that surprising in view of the fact that the integral over the boundary $B$ of the unit disc does not play any significant role in pertinence to the order of magnitude and in the absolute convergence of both the Fourier and the spherical transforms of functions in $H^{2}$. This integral could be easily suppressed without damage to the main course of the proof. Thus as far as the problems of the otder of magnitude and the absolute convergence are concerned the behavior of $\widehat{f}(\lambda, b)$ and $\widehat{f}(\lambda)$ is almost the same

REMARK 4.2. We would like to point out that the previous analysis applies almost exactly to the spherical transforms on the Lobaschowski space associated with the complex group SL(2.C) (see [7], pp. 400-402). Our treatment here will be very brief.

In this case the spherical transform is defined to be

$$
\widehat{f}(\nu)=\frac{1}{2 \nu} \int_{R} f(t) \sinh t e^{i \nu t} d t
$$

so that the transform of $f(t+h)$ is

$$
\frac{1}{2 \nu} \int_{R} f(x) \sinh (x-h) e^{2 \nu(x-h)} d x .
$$

As $h \rightarrow 0 \sinh (x-h)=O(\sinh x)$ and the transform of $f(t+h)-f(t)$ is equal to $\left(e^{-\imath \nu h}-1\right) \hat{f}|\nu|$ as usual and this leads to the final estimate

$$
\int_{\nu}^{\infty}|\widehat{f}| \nu^{2} d \nu=O\left(\nu^{-2 \alpha}\right)
$$


or equivalently

$$
\int_{\nu}^{\infty}|\widehat{f}|^{2} d \nu=O\left(\nu^{-2 \alpha-2}\right)
$$

as $\nu$ goes to infinity. The other part of the proof is very clear by now. Moreover the range of $\beta$ for which $\int_{\nu}^{\infty}|\widehat{f}|^{\beta} d \nu$ is bounded is better than that found in the case the spherical transforms for functions on $H^{2}$. In this case Hölder's inequality for $\beta \leq 2$ leads to

$$
\int_{\nu}^{\infty}|\widehat{f}|^{\beta} d \nu=O\left(\nu^{1-\alpha \beta-\frac{3 \beta}{2}}\right)
$$

which in turn gives

$$
\frac{2}{2 \alpha+3}<\beta \leq 2
$$

for the boundedness of the last quantity as $\nu \rightarrow \infty$.

\section{CONCLUDING REMARKS}

It would be convenient to end with a few comments-of a rather heuristic nature-which might cast some light on the treatment of the problem on other domains. Our first target in this respect is the group SL(2.R) denoted here by $G$ for brevity. Thus for a function $f(g) g \in G$ a small translation suitable for the Lipschitz conditions in this case is given by $f(g h), h \in G,|h| \rightarrow 0$. Thus $f$ belongs to $\operatorname{Lip}(\alpha, 2)$ on $G$ if

$$
\|f(g h)-f(g)\|_{2}=O\left(|h|^{\alpha}\right)
$$

$0<\alpha \leq 1,|h| \rightarrow 0$. With this in hand, one can apply Parseval's identity (see [4], p. 346, [6], Vol. 2, p. 53) in order to obtain the required estimates for the Fourier (spherical) transforms $\hat{f}$ corresponding to the principal and discrete series representations of $G$. The occurrence of the two integrals and the one summation on the right side of the identity causes no problem because on the one hand $\operatorname{coth} \lambda$ tends to one for large $\lambda$, thus the two integrals are treated in the same manner. On the other hand the three parts are majorized by the same quantity $O\left(|h|^{\alpha}\right)$; this enables us to deal with each part on its own. This would be easier; besides it will not affect the final conclusions.

Secondly, we hint that the previous analysis is amenable to treatment of the problem on other classical groups and their allied symmetric and homogeneous spaces (see [1], Chapter X for examples of those groups). It goes without saying that there would be some modifications in the main course of the proof when handling concrete situations such as $\operatorname{SL}(2, \mathrm{C})$ for example. Thirdly, one could explore the validity of the present line of thoughts on semi-simple Lie groups in general. A clue to investigating this problem lies in an estimate for the Harish-Chandra c-function near infinity in that case (see [2], p. 450 and [3], p. 183). These points will be taken up in a forthcoming paper.

\section{REFERENCES}

[1] HELGASON, S., Differential Geometry, Lie Groups, and Symmetric Spaces, Academic Press, New York, 1978.

[2] HELGASON, S., Groups and Geometric Analysis, Academic Press, New York, 1984.

[3] GANGOLLI, R. and VARADARAJAN, V.S., Harmonic Analysis of Spherical Functions on Real Reductive Groups, Springer Verlag New York, 1988.

[4] SUGIURA, M., Unitary Representations and Harmonic Analysis, Wiley, New York, 1975.

[5] TITCHMARSH, E.C., Theory of Fourier Integrals, Oxford University Press, 1948.

[6] WARNER, G., Harmonic Analysis on Semi-Simple Lie Groups, Vol. 2, Springer Verlag, New York, 1972.

[7] WAWRZYNCZYK, A., Group Representations and Special Functions, D. Reidel Publishing Company, Boston, 1984. 


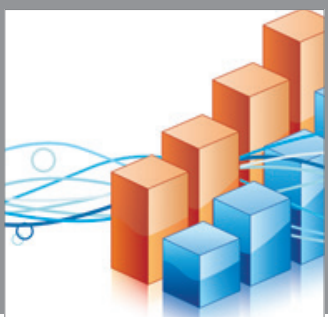

Advances in

Operations Research

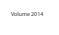

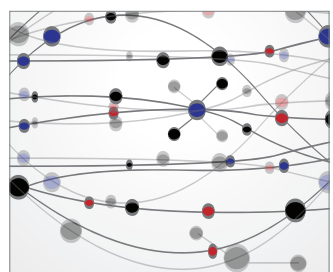

\section{The Scientific} World Journal
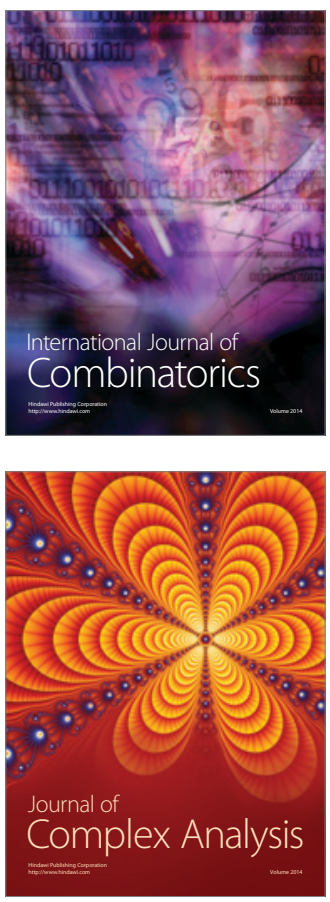

International Journal of

Mathematics and

Mathematical

Sciences
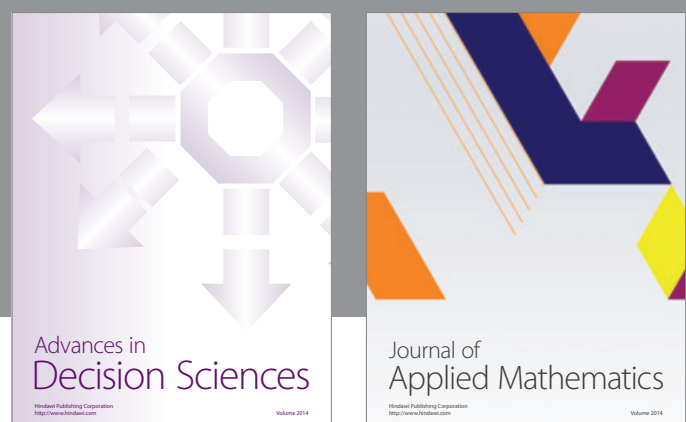

Journal of

Applied Mathematics
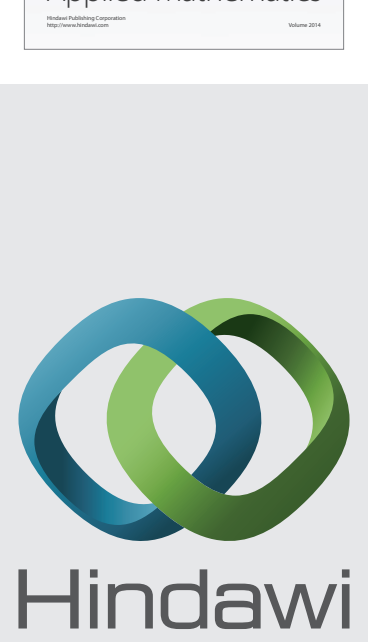

Submit your manuscripts at http://www.hindawi.com
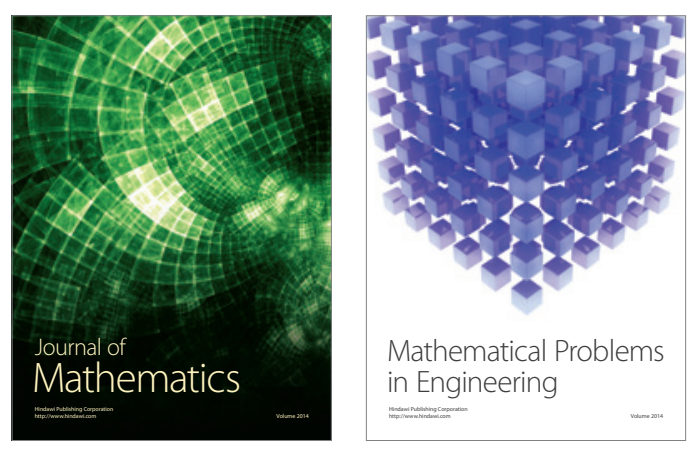

Mathematical Problems in Engineering
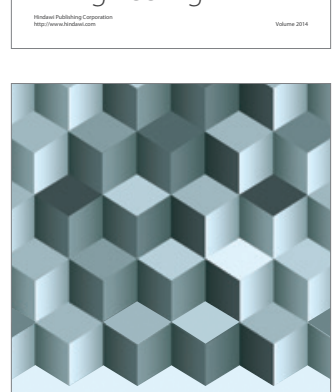

Journal of

Function Spaces
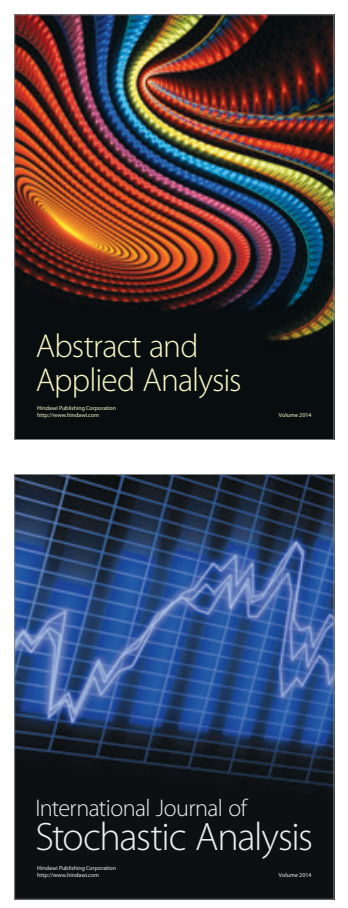

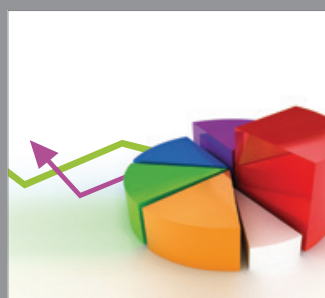

ournal of

Probability and Statistics

Promensencen
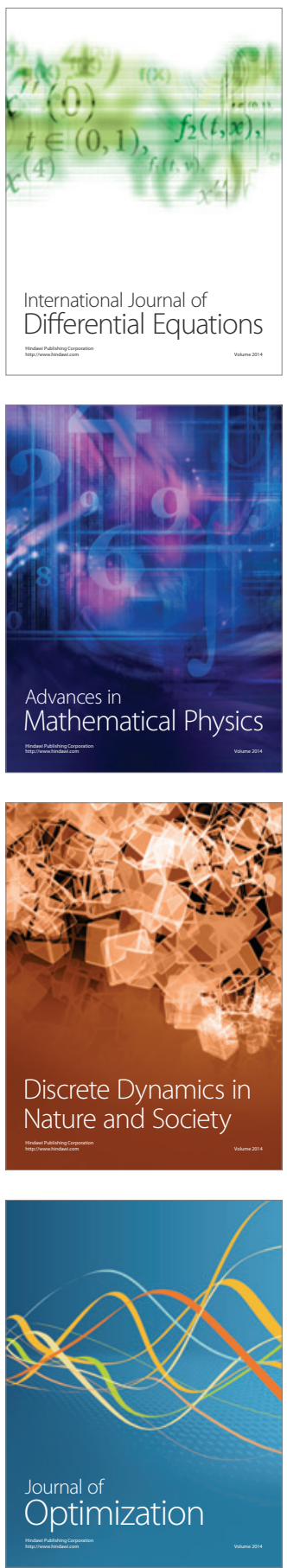